\title{
Pushing the limit of earthquake detection with distributed acoustic sensing and template matching: a case study at the Brady geothermal field
}

\author{
Zefeng Li and Zhongwen Zhan \\ Seismological Laboratory, California Institute of Technology, Pasadena, CA 91125, USA. E-mail: zefengli@gps.caltech.edu
}

Accepted 2018 August 30. Received 2018 August 22; in original form 2018 April 24

\begin{abstract}
SUMMAR Y
Template matching has been widely applied in the detection of earthquakes and other seismic events due to its power in detecting weak signals. Recent studies using synthetics have shown that application of template matching to large-N arrays can potentially detect earthquakes substantially below the noise level. Here we apply template matching to the distributed acoustic sensing (DAS) data recorded in the Brady Hot Springs geothermal field, Nevada. Using 5 catalogued events, we detect 116 events and find 68 of them well below the noise level. We confirm 112 events are true earthquakes by examining the patterns of their sensor-tosensor cross-correlation sections. This demonstrates that the combination of DAS and template matching has capability to detect microseismicity below the noise level, which is unusual for conventional seismic arrays and methods. With the updated catalogue, we observe a surge of earthquakes during the shutdown of a geothermal power plant nearby. In addition, the rapid increases in the downhole pressure record coincide with intense swarms of earthquakes. These observations show a strong correlation between the seismicity frequencies and the downhole pressure changes. Finally, we investigate several factors that may affect the detection performance and compare different strategies for spatial down-sampling, in order to provide helpful insights for future large- $\mathrm{N}$ design and data processing.
\end{abstract}

Key words: Earthquake source observations; Induced seismicity; Seismic instruments.

\section{INTRODUCTION}

Template matching is a widely used technique to detect tectonic tremor and small earthquakes (e.g. Shelly et al. 2007; Peng \& Zhao 2009). It correlates pre-determined events with continuous recordings to detect events that are similar to the templates (Gibbons \& Ringdal 2006). It has been mostly applied to seismic arrays with tens of seismometers at interstation spacing from kilometres to tens of kilometres, commonly achieving a factor of 5-10 increase in the amount of earthquake detections (Shelly et al. 2007; Peng \& Zhao 2009; Meng et al. 2013). In recent years, nodal arrays and distributed acoustic sensing (DAS) significantly increase the number and density of seismic arrays at local scale (i.e. array aperture at $1-10 \mathrm{~km}$ ). These so-called large-N arrays typically consist of hundreds to thousands of sensors with metre to hundred-metre interstation spacing. Compared to traditional arrays, large- $\mathrm{N}$ arrays provide unprecedented capability for detection of small earthquakes and other sources (Inbal et al. 2015, 2016; Riahi \& Gerstoft 2015; Li et al. 2018).

Recently, Li et al. $(2015,2018)$ performed a series of synthetic tests of template matching on the 5200-sensor Long Beach nodal array geometry. Specifically, they evaluated detection performance as a function of signal-to-noise ratio (SNR) using local M 1-2 earthquakes within $50 \mathrm{~km}$ from the array, and used synthetics to demonstrate that earthquakes remain detectable at SNR down to $10^{-5}$. This detectability for earthquakes far below noise level is rarely seen with regular seismic arrays and conventional detection methods (Li et al. 2018). Based on these findings, we ask the following questions: (1) with extremely dense arrays, can we push the limit of detection to earthquakes well below noise level in real data? (2) If such events are detected, how can we verify them? (3) Is there an optimal choice of array density, in consideration of the trade-off between detection performance and project budget? Especially, we would like to avoid excessively dense sensors while maintain desired detection performance. To answer these questions, we investigate the performance of template matching on a DAS array deployed recently in the Brady Hot Springs geothermal field in Nevada (Feigl \& PoroTomo Team 2017).

DAS is an emerging technology that draws increasing attention in exploration and earthquake seismology, due to its capability to deploy a massive number of sensors, convenient data acquisition and spatially scalable distributed measurement (Daley et al. 2013; Mateeva et al. 2013; Feigl \& PoroTomo Team 2017; Hornman 2017; Lindsey et al. 2017; Zeng et al. 2017). DAS transforms every metre 
of a telecommunication optical fibre into a linear strainmeter using an optoelectronic interrogator unit. The interrogator sends laser pulses into the fibre-optic cable and measures strain (or strain rate) along the fibre based on the distortion in backscattered light. DAS measures strain over a gauge length, whereas seismometers measure velocity/acceleration at a point. In addition, DAS data have some characteristics that are distinct from typical seismic waveforms that seismologists are familiar with (Dou et al. 2017). For example, DAS measures the linear strain or strain rate with only horizontal component along the fibre. Therefore, they have a direction-dependent sensitivity to different seismic waves, for example, $P, S$ and surface waves (Benioff 1935; Hornman 2017; Linsey et al. 2017; Wang et al. 2018). Besides, the ground coupling may not be as strong as for traditional seismometers and its effects on the fidelity of waveforms and amplitudes are still unclear (Hornman 2017; Martin et al. 2017).

Successful template matching application requires high waveform repeatability on DAS. Perfect repeatability means that events at different times but with identical source mechanisms and propagation paths should have the same instrument recordings. Biondi et al. (2017) and Martin et al. (2017) examined the recordings of two quarry blasts and an earthquake doublet on the Stanford DAS array and found their DAS waveforms have comparable repeatability to that of broad-band seismometers. On this basis, we apply template matching to two weeks of DAS data collected by the PoroTomo project in the Brady geothermal field in Nevada (Feigl \& PoroTomo Team 2017). Note that here the DAS data are strain rate with only horizontal components (along the fibre strikes). Using five catalogued earthquakes as templates, we detect many more events and find that more than half of them are hardly discerned on the DAS waveforms. We demonstrate that they are real earthquakes below noise level instead of false detections. Finally, we explore the detection performance as a function of available sensors and different spatial down-sampling strategies, in order to provide a reference for future design of DAS arrays.

\section{DATA}

The Brady Hot Springs geothermal field in northwestern Nevada (Fig. 1) is a transtensional tectonic regime with SW-NE striking normal fault systems. Recent studies show that the fault systems not only create damage zones that form the geothermal resources, but also act as conduits that link the shallow aquifers to the geothermal reservoir (Davatzes et al. 2013; Ali et al. 2016; Laboso \& Davatzes 2016; Siler et al. 2016). A geothermal power plant has been operating in this area since 1992 (Ettinger \& Brugman 1992). When the plant operates, underground superheated brine is carried up to release geothermal energy and drive the plant turbines, and then the cooled water is injected back to the subsurface.

In March 2016, the PoroTomo project (Feigl \& PoroTomo Team 2017) was carried out in this area, with the goal to characterize the reservoir porosity properties in the geothermal system. The project deployed multiple technologies and collected a variety of data sets including active seismic sources, surface seismometers, DAS, and distributed temperature sensing (DTS), pressure sensors in observation wells, GPS, and images for interferometric synthetic aperture radar (Feigl \& PoroTomo Team 2017).

The experiment included four different intentionally manipulated stages of the power plant operation. The four stages were described by Cardiff et al. (2018) and briefly summarized here: (1) March
11-13, normal operation, during which the production and injection wells were all working as normal. (2) March 14-17, shutdown, during which the power plant was halted and both production and injection stopped. (3) March 18-21, increased infield injection, during which the production and infield injection wells worked whereas far-field injection closed, which results in an increased amount of cooled water injected near the site. (4) March 22-25, resume normal operation. Readers are referred to fig. 1 in Cardiff et al. (2018) for locations of injection and production wells.

In this study, we primarily focus on the DAS array. It was deployed during 2016 March 3-26, as a 'fishbone' shape with a maximal aperture of $\sim 1.5 \mathrm{~km}$ in the NE-SW direction, buried at $0.5 \mathrm{~m}$ in depth. It uses an 'intelligent' DAS (iDAS ${ }^{\mathrm{TM}}$ ) system by Silixa Ltd, and consists of 8720 sensors with spatial sampling interval at $1 \mathrm{~m}$. The raw DAS data were recorded at $1000 \mathrm{~Hz}$ (https://gdr.openei.org/submissions/980) and resampled to $100 \mathrm{~Hz}$ (https://gdr.openei.org/submissions/993). The gauge length is $10 \mathrm{~m}$. During the PoroTomo campaign, there are five earthquakes determined by a permanent seismic network installed in 2010 in the region (Nathwani et al. 2011). These earthquakes have magnitude around 0 and are 750-1250 m below the ground surface. Fig. 1(b) shows the DAS waveforms of one of the five template earthquakes (Supporting Information Figs S1-S4 show waveforms of the other four events). We use them as templates to search for similar events in the continuous DAS data. One of the core questions we ask in this study is, with an improved catalogue, whether correlation exists between the microseismicity and the plant operation stages.

\section{METHOD}

Template matching first performs cross correlation on a sensor-tosensor basis and then stacks all individual cross correlations into a network mean (Gibbons \& Ringdal 2006). In this study, cross correlation is implemented in time domain. Prior to template matching, we perform simple preprocessing to the DAS data: remove the mean and filter the DAS waveforms at $1-15 \mathrm{~Hz}$ in order to retain the dominant frequency content of local earthquakes. Due to the coupling with the ground and the fibre orientation, the SNR varies significantly for different sensors (e.g. Fig. 1b). Therefore, we only use the recordings with SNR $>10$, which is defined as signal to noise power ratio on the bandpass filtered waveforms. The noise and signal windows are $3 \mathrm{~s}$ before and after the first arrival, respectively. We cut 6 -s waveforms starting from the event origin time to include the $P, S$ and coda waves. Note that the $P$ and $S$ waves are hardly separable due to short source-receiver distance (Fig. 1b).

After obtaining the stacked network mean cross correlation, we perform peak detection using a threshold of nine times Median Absolute Deviation (MAD) above noise level, where MAD = median $(\mid \mathrm{CC}-$ median $(\mathrm{CC}) \mid)$ and $\mathrm{CC}$ is the mean cross correlation. MAD measures the statistical fluctuation of the background noise. It is more resilient to outliers than standard deviation and has been widely used for outlier detection (e.g. Shelly et al. 2007). The detection significance of a peak can be quantified as eq. (1):

Detection Significance $=\frac{\text { Peak }- \text { Median }}{\text { MAD }}$.

By this definition, a peak with significance $\geq 9$ is considered as a positive detection. We choose 9 as a threshold because the probability of exceeding 9 MAD for a normally distributed random variable is $5.9 \mathrm{e}^{-10}$, corresponding to less than one expected false detection during the two-week study period. We then remove the duplicated detections by different templates and merge the detections that are 


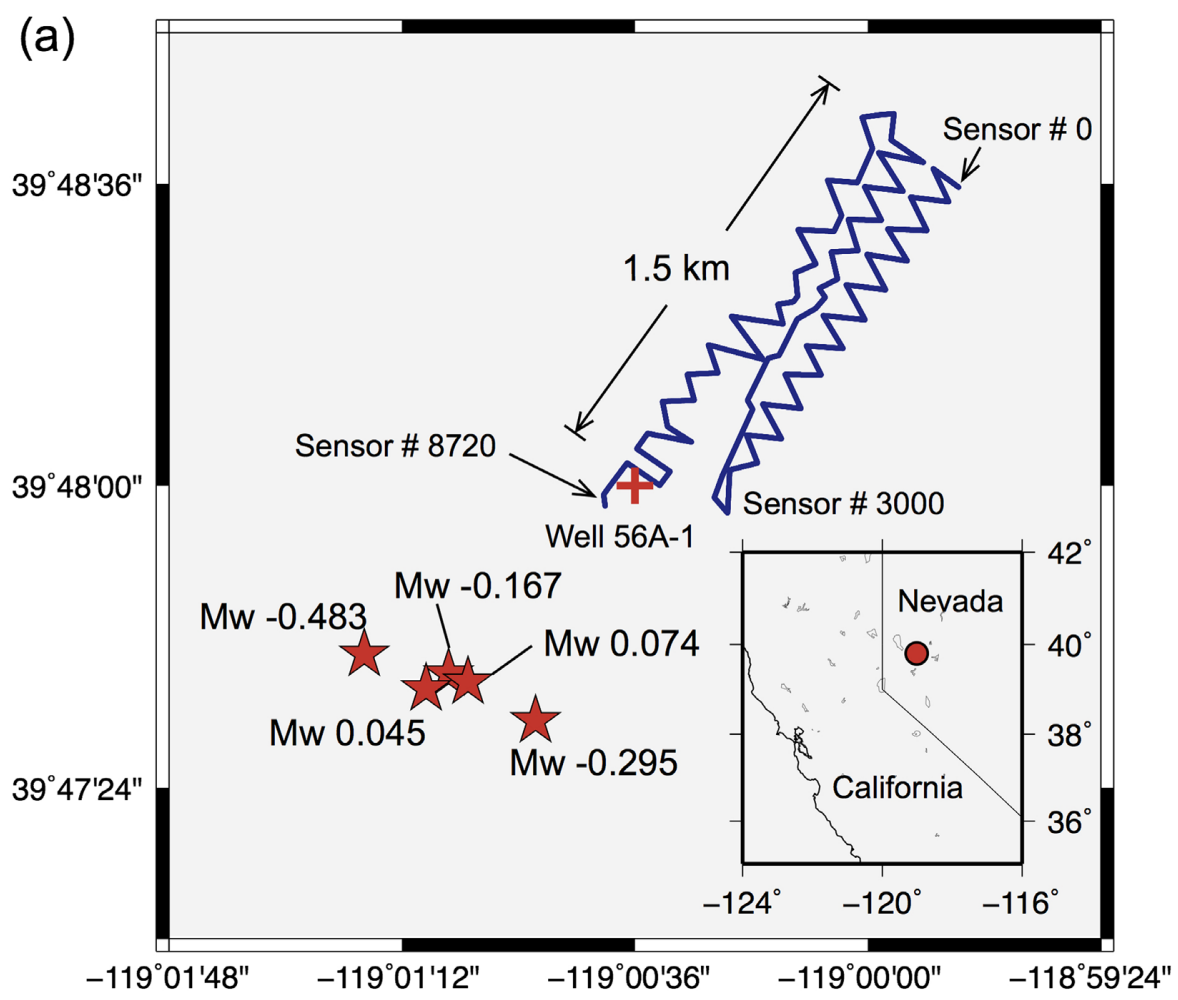

(b)

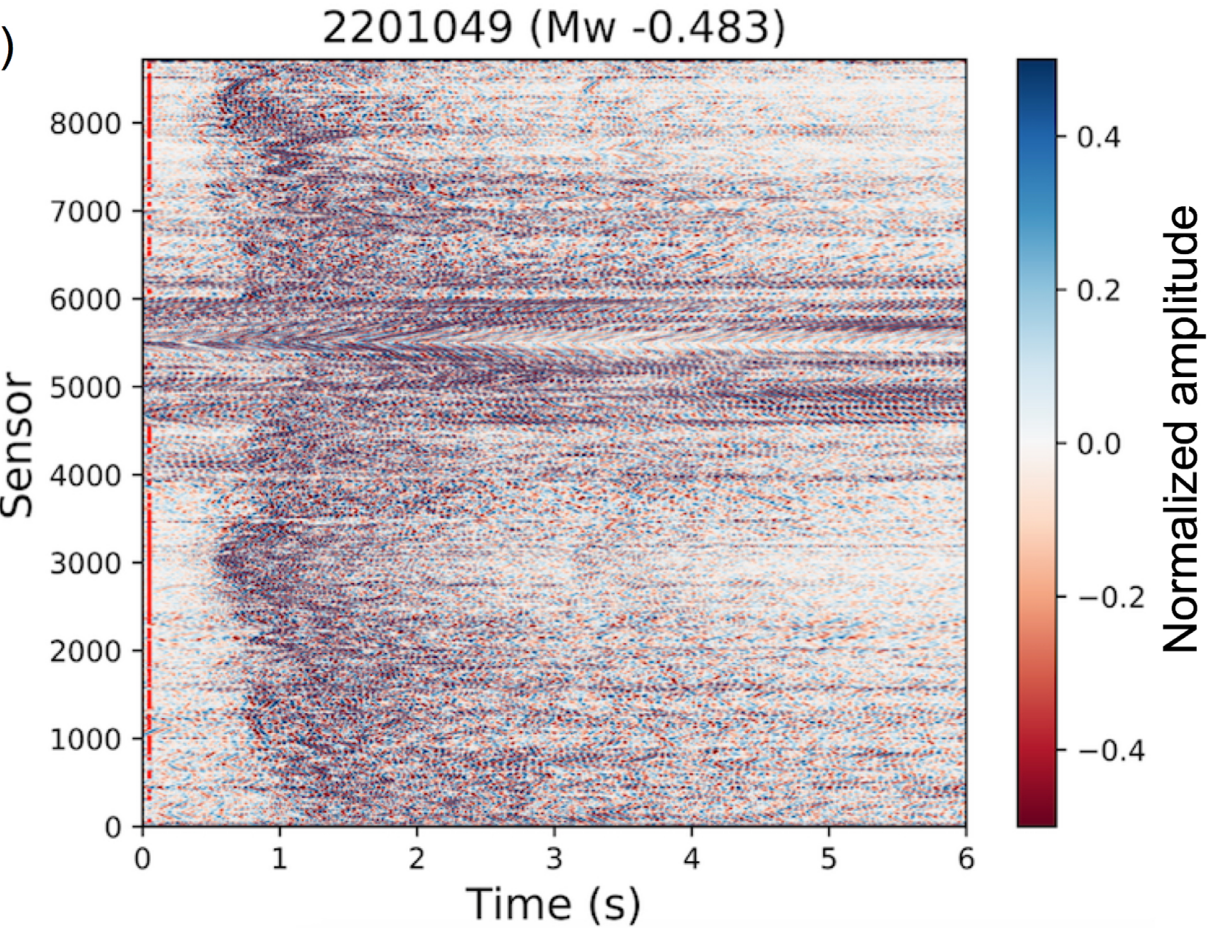

Figure 1. (a) Map of template earthquakes (red stars), distributed acoustic sensing (DAS) array (blue line) and the observation well 56A-1 (red cross) in the Brady Hot Springs geothermal field. The red dot in the inset marks the location of the Brady Hot Springs geothermal field. (b) Bandpass filtered 1-15 Hz DAS waveforms with mean removed for an M -0.483 catalogue earthquake, which occurred at $39.79442^{\circ} \mathrm{N}, 119.02162^{\circ} \mathrm{W}$ with depth at $426 \mathrm{~m}$ above sea level, on UTC 08:36:26.61, 2016 March 14. The red dots on the left indicate the sensors that are included in template matching (signal-to-noise ratio $>10$ ). Each trace on individual sensors is normalized by the maximum of that trace. The colour shows the amplitude after normalization and is clipped at [ $-0.5,0.5]$. 

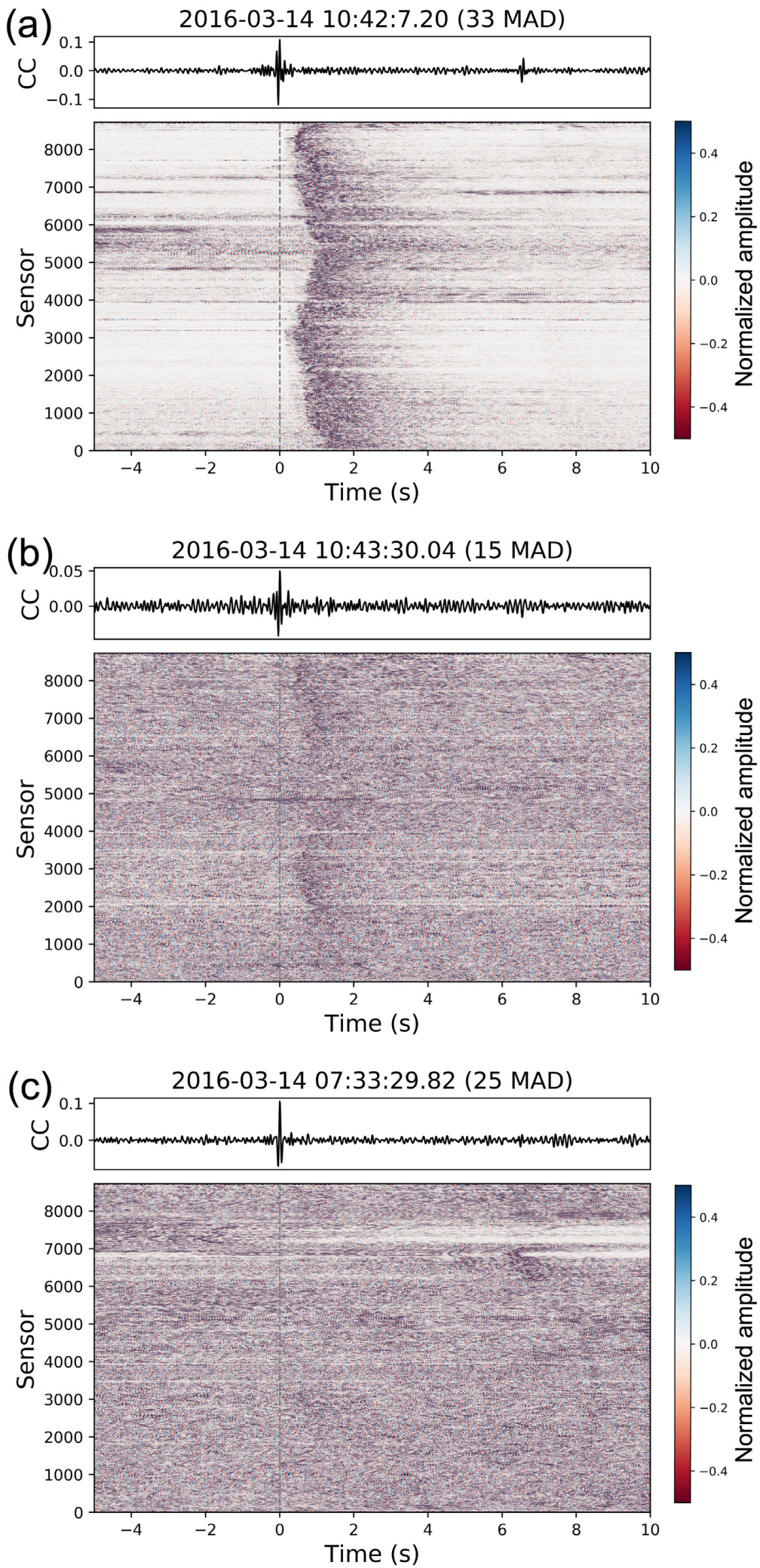

Figure 2. DAS waveforms of representative examples at different levels of visibility. From top to bottom are: (a) visible on most of the sensors; (b) visible on part of the sensors; (c) invisible on almost all the sensors. The black curves in the upper panels are the mean stack of sensor-to-sensor cross correlations after time-shifting to the origin time. The black vertical dashed lines mark the event origin times. Note that each waveform on individual sensors is normalized by its maximum. 


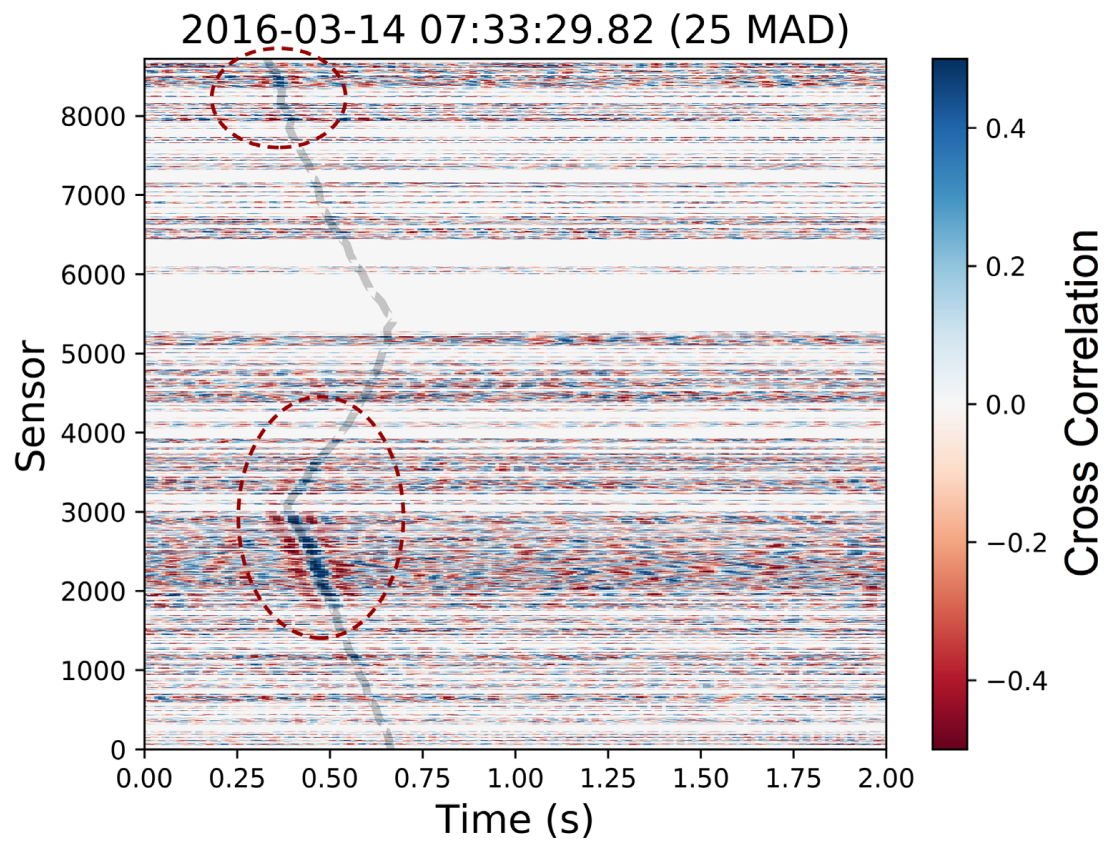

Figure 3. Cross-correlation section of a high-significance low-SNR detection (Fig. 2c) before time shifting. Sensors excluded in template matching are left blank. The dashed gray line marks the predicted arrivals of the template event, assuming a homogenous velocity of $4 \mathrm{~km} \mathrm{~s}^{-1}$. The red circles mark the sensors which have the highest cross-correlation values. Each cross correlation on individual sensors is normalized by its maximum.
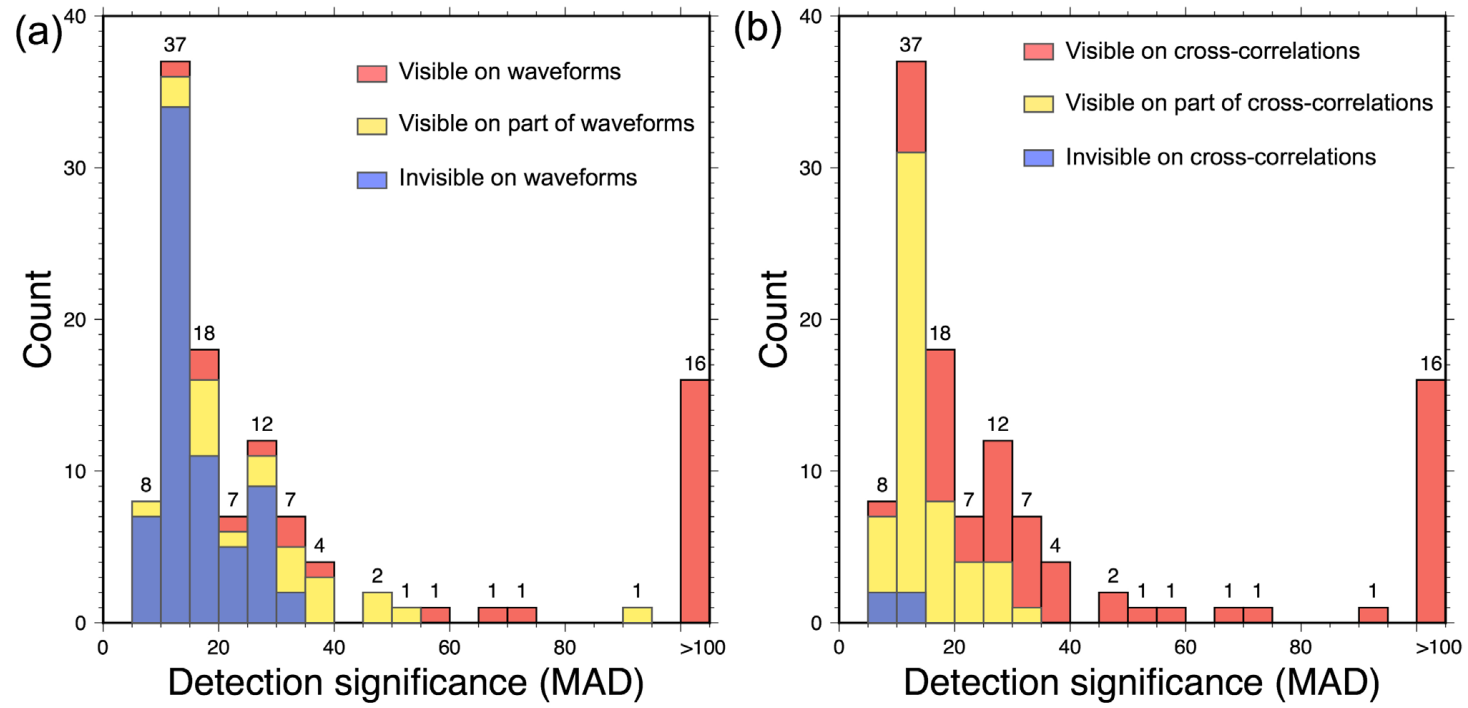

Figure 4. Histograms of detection significance at three levels of visibility on (a) DAS waveforms and (b) cross correlations. The bar in each bin is stacked from bottom to top with the numbers of events in 'visible' (blue), 'visible on part' (yellow) and 'invisible' (red) levels. The number annotated above a bin is the count of all the detections in that bin. Note that most the detections invisible on DAS waveforms become visible or partly visible on cross correlations.

less than $3 \mathrm{~s}$ apart. Due to the poor azimuth coverage (Fig. 1a), we do not relocate the newly detected events in this study.

\section{RESULTS}

\subsection{Detection results and validation}

Template matching produces 116 detections with the threshold at 9 MAD (Supporting Information Table S1). To check the detection quality, we plot out the waveform sections for each detection. Then we visually inspect all the waveform sections, and categorize the detections into three levels of visibility: SNR $>1$ on most of the sensors ('visible'), SNR > 1 on part of the sensors ('visible on part', usually on those close to the source), $\mathrm{SNR}<1$ on almost all the sensors ('invisible'). Fig. 2 shows representative examples for each level. After the classification, there are 21, 27 and 68 detections belonging to the 'visible', 'visible on part' and 'invisible' class, respectively (see Supporting Information Table S1 for the waveform visibility class for each event).

It is somewhat surprising that more than a half of the detections are totally invisible on the DAS waveforms. As noted above, we expect less than one false detection with the threshold at 9 MAD, which suggests most of the detections should be real earthquakes. To validate the detections, we check the pattern of cross correlations on 

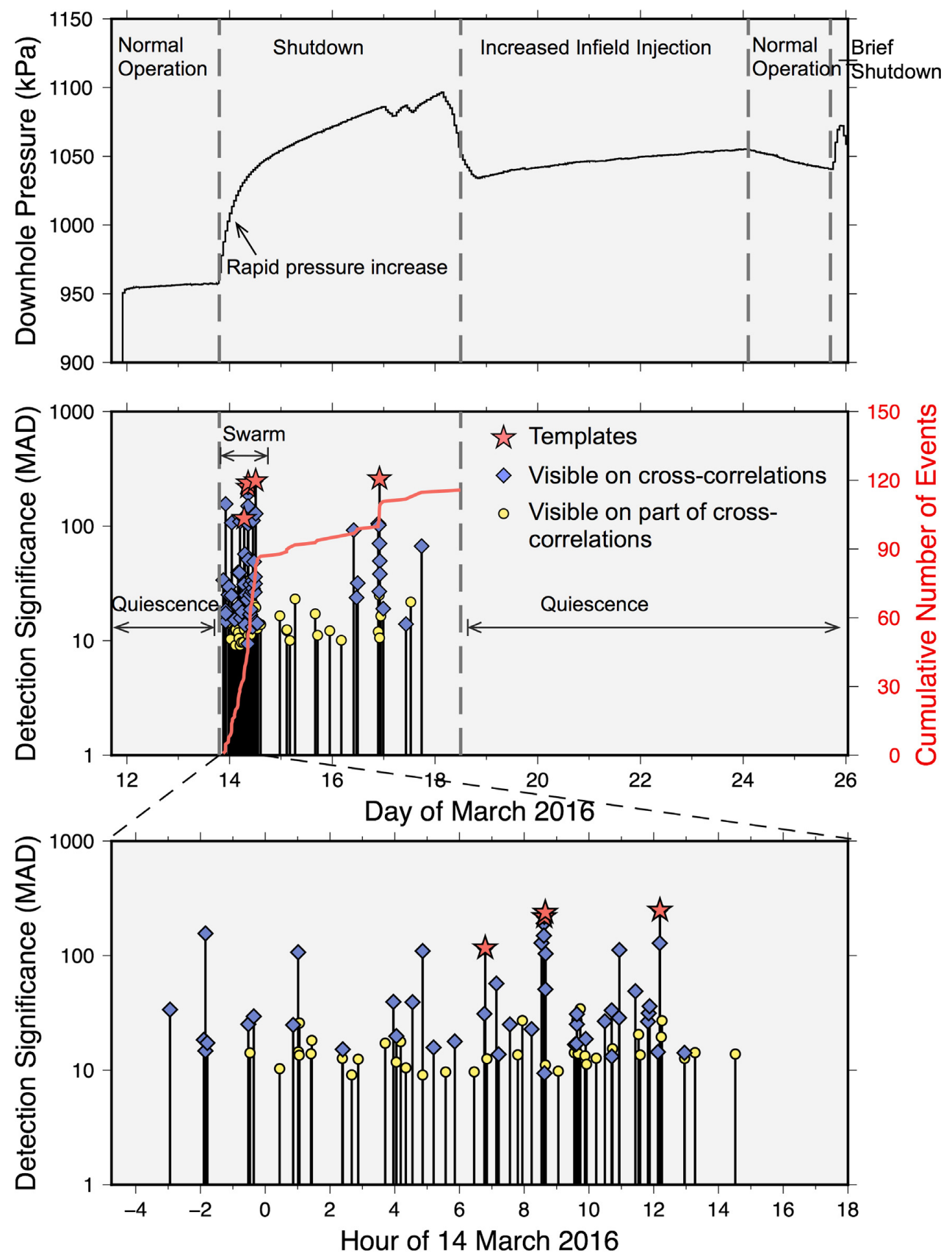

Figure 5. Earthquake sequence detected by template matching and comparison with downhole pressure measurement. (a) Downhole pressure measurement at the observation well 56A-1. The dashed grey vertical lines divide different operation stages. (b) Earthquake sequence during the two-week DAS deployment period. Stars, diamonds, and circles are template events, newly detected events visible and partly visible on cross correlations, respectively. The red curve represents the cumulative number of events over time. (c) Zoom-in plot of the earthquake swarm in (b).

individual sensors. We plot out the sensor-sensor cross-correlation sections similar to those of waveforms, and check the amplitude and moveouts of the high cross-correlation values (Fig. 3). As Fig. 3 shows, although invisible on DAS waveforms, the events can be revealed from their cross-correlation sections. The sensors with the shortest distance to template events (i.e. sensors around 3000 or 8720 as marked in Fig. 1) have the highest cross-correlation values, compared to the sensors that are farther away. In addition, the timings of the peaks are consistent with predicted travel times from template event locations (Fig. 3). These suggest that the detected events originated from locations near or identical to the template events.

With these features in mind, we visually examine the images of cross correlations for each detected event. We categorize the visibility level on cross correlations, following the same classification criteria for waveform images. We find 61, 51 and 4 events on cross correlations belong to 'visible', 'visible on part' and 'invisible' levels, respectively (see Supporting Information Fig. S5 for representative examples; see Supporting Information Table S1 for the cross-correlation visibility class for each event). This suggests 


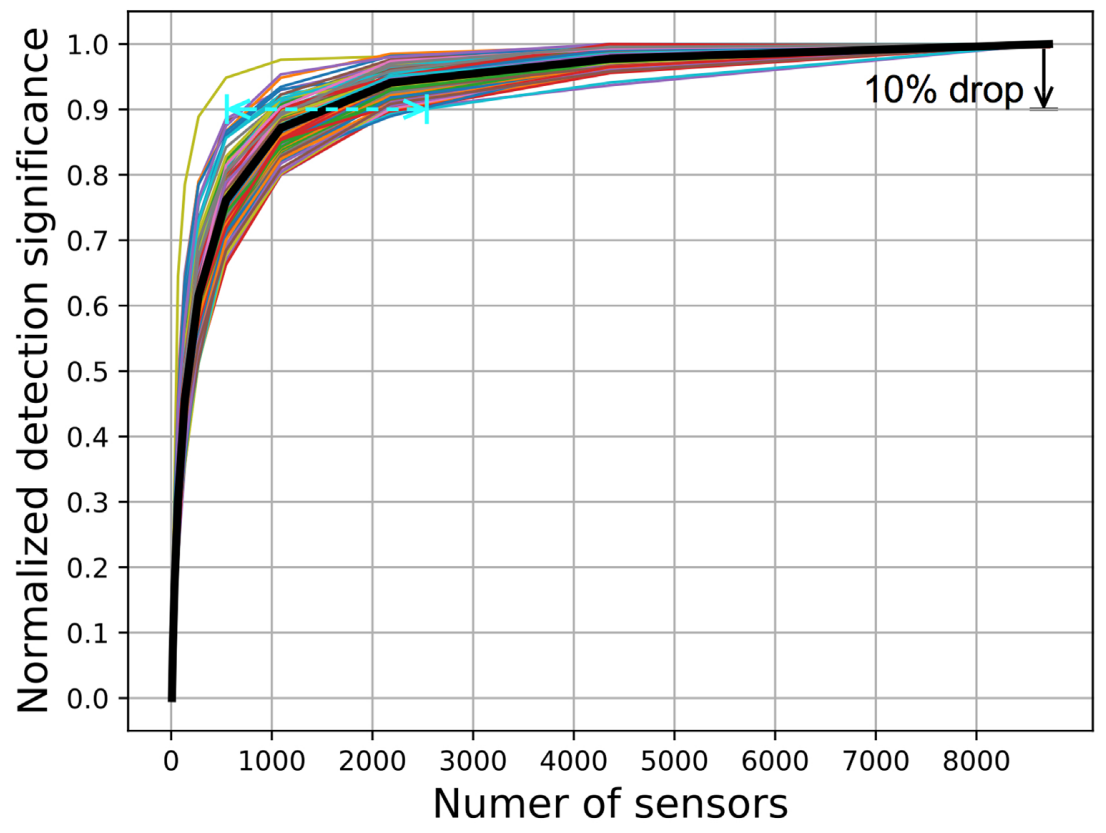

Figure 6. Normalized detection significance, defined as (Peak-Median)/MAD and normalized by the event maximum and minimum, as a function of sensor number. Each coloured curve represents the normalized detection significance of an event using different numbers of sensors. At a given number of sensors, we bootstrap 100 times and measure their average peak significance. The thick black curve represents the mean of the 112 events. The cyan dash bar marks the range of the number of sensors needed to achieve significance level at 90 per cent of that using the entire array.

96.6 per cent of the events are visible at least on part of their cross correlations, in comparison to only 41.4 per cent on the DAS waveforms. Only four of the newly detected events are invisible on cross correlations and thus cannot be verified in this way. These events are excluded in the later discussions. Fig. 4 shows that overall the visibility on cross correlations is significantly enhanced compared to DAS waveforms. In particular, there are 64 events that have DAS waveforms below noise level but become visible on their cross correlations.

\subsection{Earthquake sequence}

By plotting the verified events with time, we observe a striking correlation between the seismicity and the power plant operation stages (Fig. 5). All the earthquakes occurred within the shutdown period of the geothermal power plant, in stark contrast with the quiescence before and after. The concentration of seismicity during the shutdown period also serves as strong evidence that these detections are real earthquakes controlled by the undergoing shutdown process, given that the background noise level and instrument sensitivity remained stable throughout the operation stages. If these detections were caused by random noise fluctuations, they would have been distributed over the study period (336 hr), rather than concentrated in the relatively short shutdown period ( $5 \mathrm{hr}$ ). By comparing the event timings with the vibroseis experiments during the PoroTomo Project, we also rule out the possibility that the vibroseis signals are falsely detected as 'earthquakes' (Supporting Information Fig. S6). Therefore, they are more likely controlled by a physical mechanism: during the shutdown, the extraction cessation leads to the decrease in effective stress on faults, which favours the occurrence of seismicity (Cardiff et al. 2018).

The shutdown began at 2016 March 13 19:15 UTC, and the earliest event identified from this study occurred $2 \mathrm{hr}$ later at 21:03 UTC (Fig. 5c), which was $9.5 \mathrm{hr}$ earlier than the first catalogued event.
This suggests faster propagation speed of pressure/stress change than what was expected from the original catalogue. At the beginning of the shutdown, the downhole pressure at Well 56A-1 increased rapidly and an intense earthquake swarm with 85 events occurred (Figs $5 \mathrm{a}$ and b). The swarm lasted for about $18 \mathrm{hr}$. As the increase of downhole pressure slowed down, the seismicity became less and less active. In the increased injection stage, although the pressure maintains at a relatively high level, there is no seismicity observed.

\section{DISCUSSION}

\subsection{Temporal evolution of seismicity and control mechanism}

We applied template matching to detect small earthquakes in the Brady geothermal field, using two-week data recorded by the 8720 sensor DAS array. Based on the five catalogued events, we detected 116 events, 112 of which can be verified as real earthquakes from their cross-correlation sections. It is noteworthy that more than a half of them are below noise level. Cardiff et al. (2018) compared the long-term and short-term earthquake catalogues with pumping record and found that most of the earthquakes in the Brady geothermal field occurred during the shutdowns in pumping. With more small earthquakes detected in this study, we confirm this correlation much more confidently (Fig. 5). The quiescence in the normal operation and the sudden surge of seismicity during the plant shutdown supports the hypothesis that during pumping cessation the effective stress on faults decreases and microseismicity tends to occur (Cardiff et al. 2018).

With a more complete catalogue, we observe more details in the temporal evolution of the seismicity, which is out of the resolution of the original catalogue. The shutdown first triggered an intense swarm that contains 85 events, which coincided with rapid 


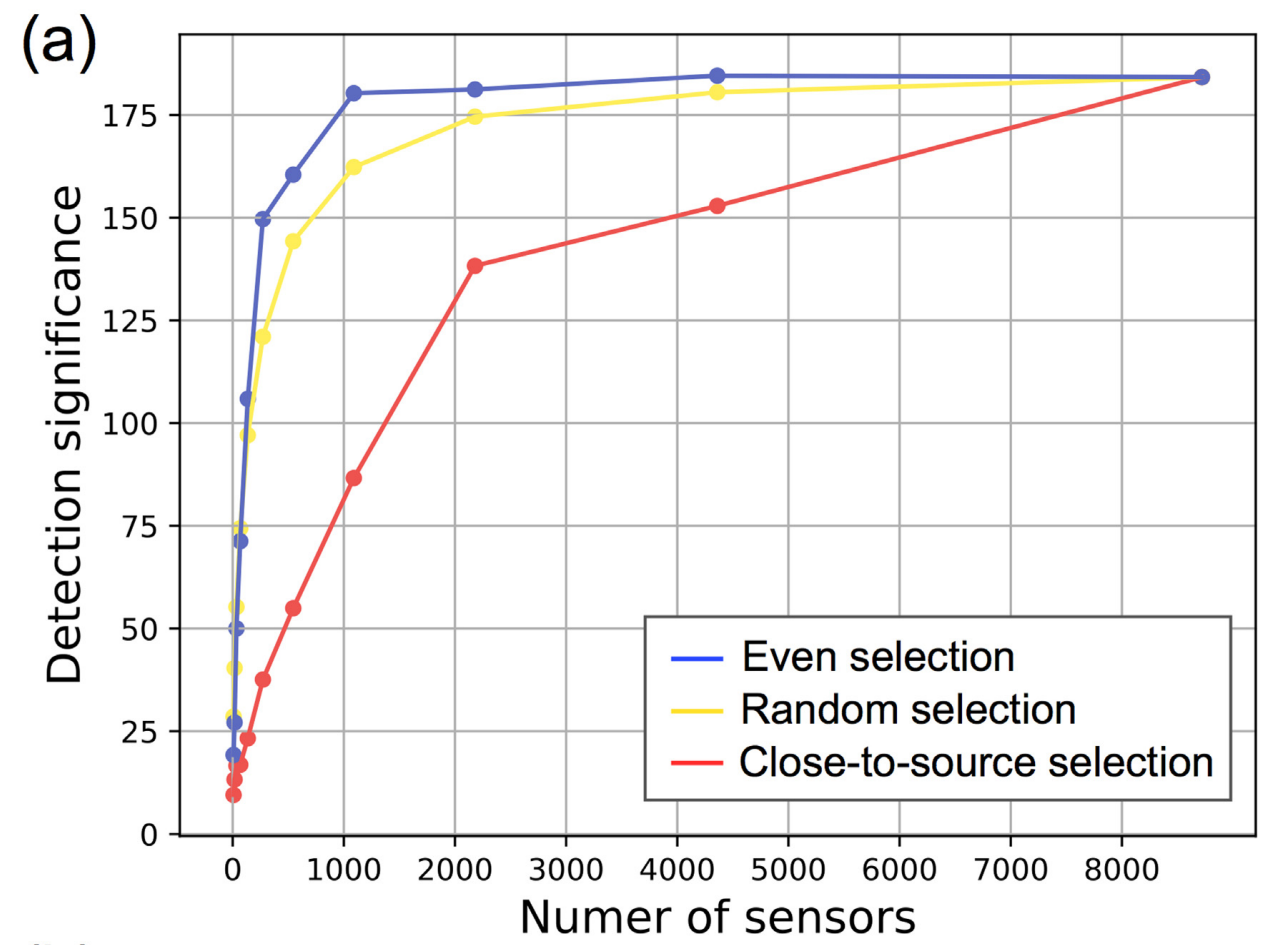

(b)

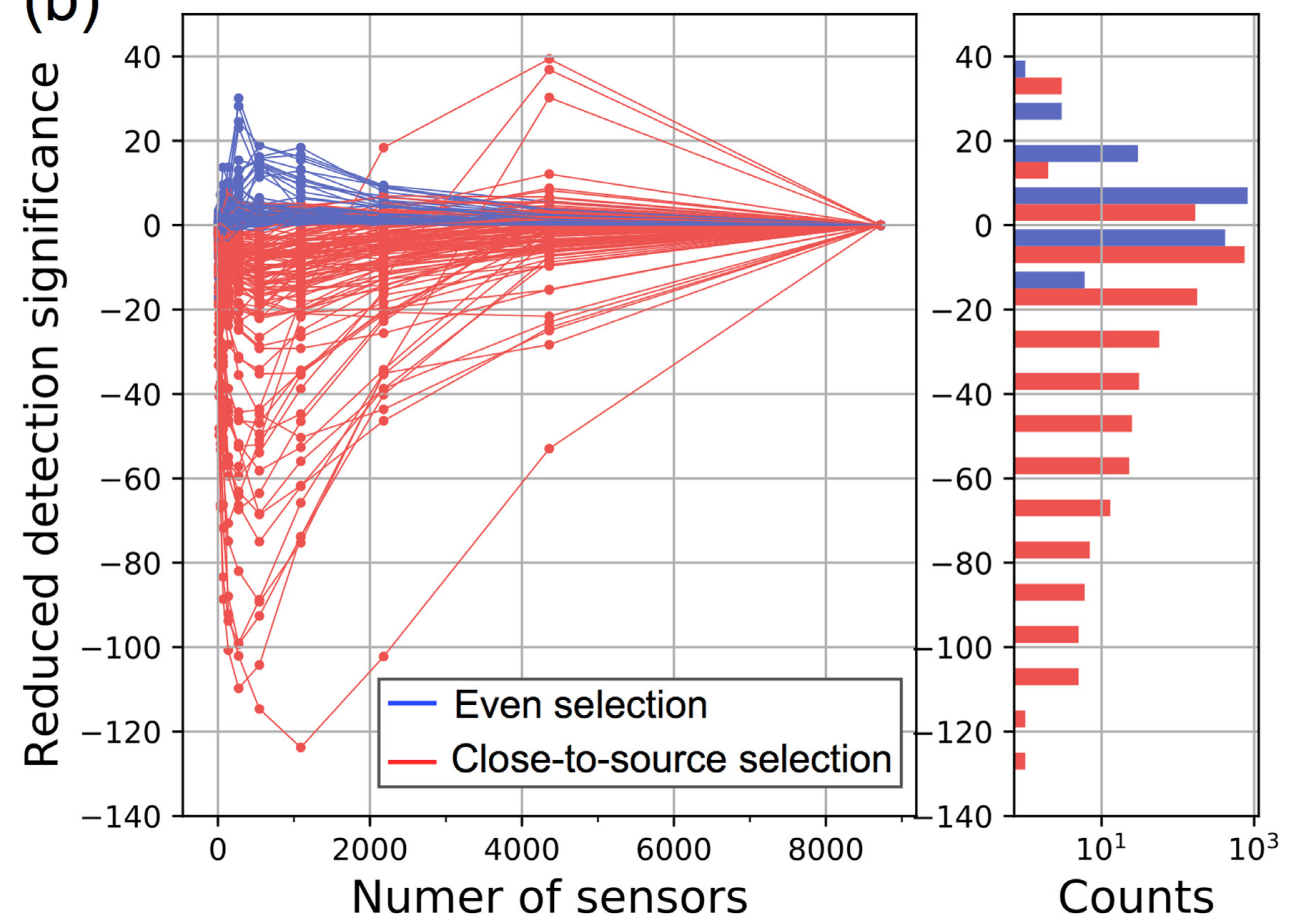

Figure 7. Detection significance ((Peak-median)/MAD) versus the number of sensors using three different sensor selections. (a) Detection significance for an example event (UTC 03/13/2016, 22:09:08.25) using three different sensor selections. (b) Left: reduced detection significance (with detection significance from random selection subtracted) versus the number of sensors for all the 112 events. Right: histograms of reduced detection significance for even selection (blue) and close-to-source selection (red).

increase of the downhole pressure at the beginning (Fig. 5). After the swarm, the pressure increased gently and the seismicity became less frequent. However, the sudden changes in the pressure ('kicks' near the hour of 17 in Fig. 5a) also coincided with abrupt occurrence of earthquakes. Therefore, there exists a strong corre- lation between the cumulative number of events with the downhole pressure (Fig. 5). Brodsky \& Lajoie (2013) identified a similar correlation between the seismicity rate and the net production rate, using long-term (years) records in the Salton Sea geothermal field. Here we provide a strong case in short-term correlation (hours to 
(a)

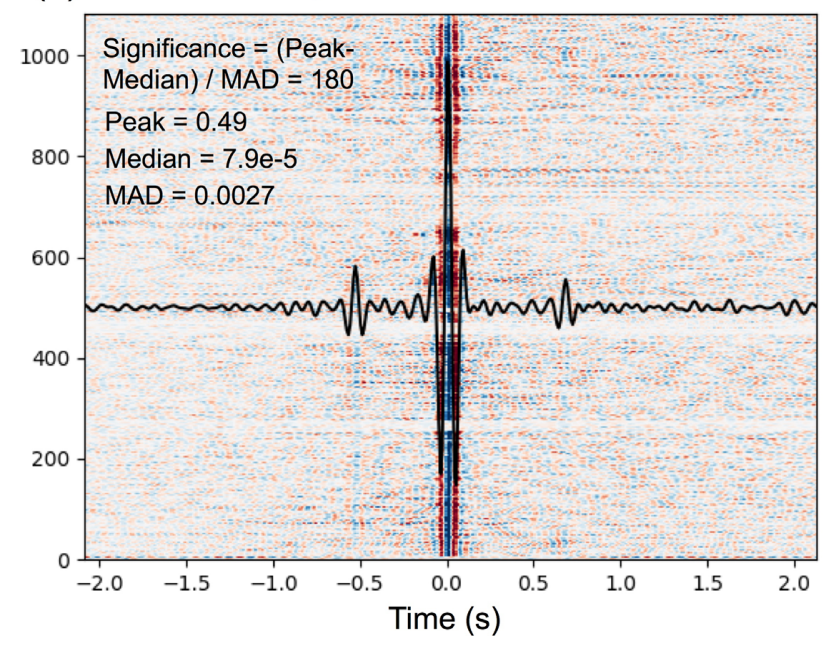

(b)

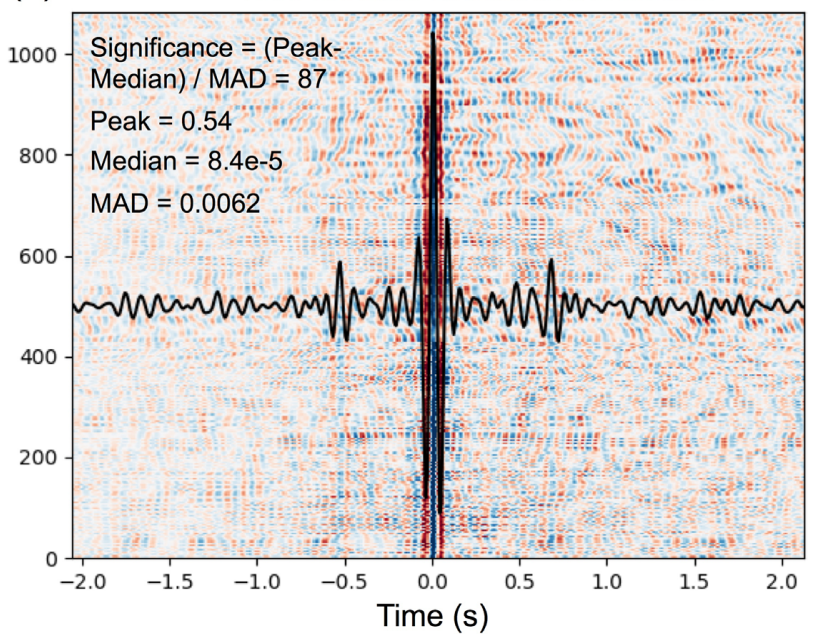

Figure 8. Cross-correlation sections for 1090 sensors (a) evenly selected and (b) close to the source using the example event in Fig. 7(a). The black line represents the stacked mean cross correlation of the 1090 individual traces. The mean trace is placed at $Y=500$ and the amplitude is exaggerated by 1000 times in order to visualize the noise fluctuation. Note the coherent stripes in (b) and the resulting fluctuation is greater than that in (a).

days). These observations suggest that the seismicity is likely controlled by the pressure change rate, rather than the absolute pressure. This agrees with the critical pressure theory in which a fault ruptures immediately when the normal stress change associated with fluid movement exceeds the criticality (Shapiro et al. 2005; Shapiro 2015).

\subsection{Detection capability of DAS arrays and control factors}

As a relatively new technology for seismic data acquisition, a DAS array can provide thousands of sensors with spacing at metres. One of the interesting questions here is the detection capability of DAS in comparison to conventional arrays. In this study, we obtained 112 verified events in the two-week DAS deployment, which is 22 times of the catalogued earthquakes. This is an unusually high factor of increase compared to 5-10 for typical template matching applications in conventional arrays (Shelly et al. 2007; Peng et al. 2009; Meng et al. 2013), which benefits from the large number of available sensors and ultra-dense spacing. Other factors, such as that the DAS array is closer to the sources than the regional array, can also contribute to the detection improvement.

Accumulating evidence has shown that large- $\mathrm{N}$ arrays enable us to robustly push the detection limit to events below noise level. $\mathrm{Li}$ et al. $(2015,2018)$ demonstrated this capability with synthetics, and this study provides a real-world example with a DAS array. Although 58.2 per cent of the events are invisible on their waveform sections, we show that almost all of them can be manifested from the cross-correlation sections (e.g. Figs 3 and 4). This is because that template matching acts as an optimal filter to maximize SNR if the target matches the template well (Turin, 1960). The stacking of the cross correlations from a large number of sensors further boosts up the peak significance on the final network trace, enabling the detection of weak signals with high confidence.

Since the five catalogued earthquakes are from -0.48 to $0.07 M_{\mathrm{w}}$, the sizes of the newly detected events are expected to be comparable or smaller, given the fact that they are missing from the original catalogue. Li et al. (2018) has shown that the detection limit using template matching on the 5200-sensor Long Beach array can reach SNR as low as $10^{-5}$. If we assume the same order of detectability for the PoroTomo 8720-senosr DAS array and a reference event with $M_{\mathrm{w}}=0$ and SNR $=10$, by simple amplitude scaling (Li et al. 2018), the smallest detectable earthquake is estimated to be $M_{\mathrm{w}} \sim$ -3 . However, for these extremely small events, determination of the size or amplitude is difficult, due to the difficulty in separating actual event waveforms from noise. The investigation of this problem will be a subject of future work.

Such powerful detectability could be compromised by imperfect similarity between the templates and the target events. Fig. 4(a) shows some events can have relatively low detection significance but good SNR if the waveform similarity is low (e.g. Fig. 2b) and vice versa (e.g. Fig. 2c). This is because detection significance depends not only on SNR but also on waveform similarity. In our detection, the visible events can have significance as low as 13 MAD while the totally invisible events can have significance as high as 35 MAD (Fig. 4a). Template matching is advantageous in detection of events with high similarity, for which the detectable events can be far below noise.

The detectability also depends on the number of available sensors. To quantify their relationship, we measure the peak significance of the 112 detected events using various numbers of sensors (8720, $4360,2180, \ldots$, decreasing by a factor of 2 in each step). For a given number of sensors, we bootstrapped 100 samples of sensor selection and average the peak significance (Fig. 6). It is expected that, in general, the peak significances increase with the number of sensors. However, we find that the improvement becomes marginal when the number of sensors exceeds a certain threshold (Fig. 6). We consider the number of sensors needed to achieve significance level at 90 per cent of that using the entire array ( $Y=0.9$ on Fig. 6). The mean curve shows that only $\sim 1500$ sensors (i.e. one-sixth of the array) on average are needed. For individual events, the number of sensors needed ranges from 500 to 2500 (Fig. 6), which are also fractions of all the sensors.

Such a ' $\Gamma$ ' curve of performance for the Brady DAS array is fundamentally different from that for the Long Beach array, which is approximately $\operatorname{sqrt}(N)$, where $N$ is the number of sensors (fig. $5 \mathrm{a}$ in Li et al. 2018). These two distinct patterns could be a result of different noise coherency on two arrays. The Long Beach array has spacing at $100 \mathrm{~m}$, which is large enough to make the noise relatively 
independent on neighbouring stations. In this case, the stacking gain generally follows sqrt(N). However, the Brady DAS array has only 1-m spacing so that the noise on neighbouring sensors are dependent. Reducing these dependent recordings would not change the diversity of the recordings, which leads to similar detection performance ( $N \sim 1500-8720$ in Fig. 6). As the decimation continues, when the remaining sensors have mostly independent noise (i.e. spacing is large enough), the detection performance starts to decrease significantly ( $N \sim 0-1500$ in Fig. 6). This pattern should be taken into account in large- $N$ design. Especially, considering the trade-off between detection performance and the economy (array deployment, data storage and computation cost), it would be ideal to set the array at the turning point of the ' $\Gamma$ ' curve.

\subsection{Optimal strategies for spatial down-sampling}

In the previous section, we discussed the dependence of detection performance on array density, which is for array design consideration. In this section, we consider a different scenario: if an array is given, what is the best way to spatially down-sample the sensors? Since large- $N$ arrays can contain thousands of sensors which makes computation costly, one may prefer to spatially down-sample an array while maintain comparable performance to that using the entire array.

In Section 3, we selected the sensors with SNR > 10, which is a common practice inherited from previous template matching applications to conventional seismic arrays (Shelly et al. 2007; Peng et al. 2009; Meng et al. 2013). This seems a natural choice because the higher quality recordings can better match targeted signals (i.e. obtain higher cross-correlation values). However, this may not be necessarily the optimal approach for dense DAS arrays. Because coherent noise on neighbouring DAS sensors can increase the MAD value, thus reducing the detection significance. In the following, we illustrate this point in details by testing three spatial sensor downsampling strategies: (1) random selection with 100 repetitions, after which the average is taken. This is to approximate the expectance of detection performance by random selection, which serves as the baseline for the other two approaches; (2) evenly down-sampling the array; (3) choosing sensors close to the sources. Note that choosing sensors close to the sources is comparable to choosing with best SNRs.

With three different selections, we run similar detection performance tests as in Fig. 6, with an example event at UTC 03/13/2016, 22:09:08.25. It turns out the even sensor selection approach results in higher detection significance than the other two in a wide range of sensor numbers (Fig. 7a). For instance, given 1090 sensors (decimation factor 8), the even selection has detection significance of 180 , whereas the close-to-source selection has detection significance of 86. Although the close-to-source selection has a higher peak crosscorrelation value $(0.054<0.047)$, its MAD value is much greater $(0.0062>0.026)$. This results in a lower detection significance, as defined in eq. (1). We observe this pattern (even selection $>$ random selection $>$ close-to-source selection) are common for most of the events, with only a few exceptions (Fig. 7b).

We interpret the high MAD value for the close-to-source selection as a result of highly coherent noise. Close-to-source selection tends to use spatially clustered sensors which share similar background noise. This leads to similar noise background cross correlations (Fig. 8), because the template waveforms on these sensors are also similar. Hence, when the individual cross correlations are stacked, the noise background cannot be effectively damped, leading to a high MAD (Fig. 8). If the number of sensors is fixed, even selection maximizes the spacing among the sensors and thus reduces the noise coherence. In other words, it preserves the diversity of the original recordings. Random selection avoids spatial sensor concentration but does not maximize the interstation spacing, which on average performs slightly worse than even selection. A few exceptions in Fig. 7(b) (close-to-source selection $>$ random selection or even selection) are likely because the gain in the peak cross-correlation value caused by good SNRs is greater than the loss in MAD caused by sensor clustering.

Considering that the presented detection results are based on the selection of best-SNR sensors, we re-run the detection with 1500 spatially evenly selected sensors. This new selection detects six more events that are previously below the 9-MAD threshold, which however do not change the observed patterns. Nevertheless, our test suggests that even sensor selection is likely the optimal choice for earthquake detection, when arrays are dense enough to have coherent noise. This finding also underscores the significance of noise coherence for large- $\mathrm{N}$ arrays (spacing at metres to tens of metres), which is widely considered as negligible for conventional seismic arrays (spacing at kilometres or longer).

\section{CONCLUSIONS}

We apply the template matching technique to detect earthquakes on DAS data in the Brady geothermal field and identify 112 earthquakes with 5 catalogued earthquakes. Although more than a half of the events have no waveforms standing out of the background noise, the visibility can be greatly enhanced on cross correlations. The amplitude and timing characteristics of the cross-correlation sections suggest that they have similar locations to those of the templates. Our study demonstrates that the combination of DAS and template matching can be used to detect microseismicity well below the noise level, which is unusual compared to conventional arrays and detection methods. In addition, we show that there exists a threshold for the number of sensors beyond which detectability enhancement becomes marginal because the noise becomes coherent and the independence of recordings decreases. On this basis, we find that for arrays with very close spacing, evenly selecting the sensors could be the optimal strategy for spatial down-sampling, because it maximizes the interstation spacing and preserves more independent recordings. These findings provide helpful instructions for future large- $\mathrm{N}$ design and data processing.

\section{ACKNOWLEDGEMENTS}

We are grateful to the Editor Jörg Renner, Nate Lindsey and the other anonymous reviewer for their helpful comments. We thank Kurt L. Feigl and the PoroTomo Team for making the DAS data and the downhole pressure record publically available. They can be accessed on the Geothermal Data Repository (https://gdr.openei.o rg/, keyword: PoroTomo, last accessed on 2018 June 20). We thank Xiangfang Zeng for helpful discussions on data processing. This work is partially supported by the Nation Science Foundation Grant 1722879 and the Caltech Discovery Fund, and the President's and Director's Fund. The PoroTomo experiment in 2016 is supported by award DE-EE0006760 from the Geothermal Technologies Office of the U.S. Department of Energy. 


\section{REFER E N C E}

Ali, S.T. et al., 2016. Time-series analysis of surface deformation at Brady Hot Springs geothermal field (Nevada) using interferometric synthetic aperture radar, Geothermics, 61, 114-120.

Benioff, H. 1935. A linear strain seismograph, Bull. seism. Soc. Am., 25, 283-309.

Biondi, B.L., Martin, E.R., Cole, S., Karrenbach, M. \& Lindsey, N. 2017. Earthquakes analysis using data recorded by the Stanford DAS array, in $87^{\text {th }}$ Annual International Meeting, SEG Expanded Abstracts, 2752-2756.

Brodsky, E.E. \& Lajoie, L.J. 2013. Anthropogenic seismicity rates and operational parameters at the Salton Sea geothermal field, Science, 341, 543-546.

Cardiff, M. et al., 2018. Geothermal production and reduced seismicity: correlation and proposed mechanism, Earth planet. Sci. Lett., 482, 470477.

Daley, T.M., Freifeld, B.M., Ajo-Franklin, J., Dou, S., Pevzner, R., Shulakova, V. \& Lueth, S. 2013. Field testing of fiber-optic distributed acoustic sensing (DAS) for subsurface seismic monitoring, Leading Edge, 32, 936-942.

Davatzes, N.C., Feigl, K.L., Mellors, R.J., Foxall, W., Wang, H.F. \& Drakos, P., 2013. Preliminary investigation of reservoir dynamics monitored through combined surface deformation and micro-earthquake activity: Brady's geothermal field, Nevada (Sgp-Tr-198), in 38th Workshop on Geothermal Reservoir Engineering, Stanford University, Stanford, CA.

Dou, S., Lindsey, N.J., Wagner, A., Daley, T.M., Freifeld, B.M., Robertson, M. \& Ajo-Franklin, J.B. 2017. Distributed acoustic sensing for seismic monitoring of the near surface: a traffic-noise interferometry case study, Sci. Rep., 7, 11620.

Ettinger, T. \& Brugman, J. 1992. Brady Hot Springs geothermal power plant, in 14th New Zealand Geothermal Workshop, New Zealand, pp. 89-91.

Feigl, K.L.M., PoroTomo Team 2017. Overview and preliminary results from the PoroTomo project at Brady Hot Springs, Nevada: poroelastic tomography by adjoint inverse modeling of data from seismology, geodesy, and hydrology, in 42nd Workshop on Geothermal Reservoir Engineering, Stanford University, Stanford, CA.

Gibbons, S.J. \& Ringdal, F. 2006. The detection of low magnitude seismic events using array-based waveform correlation, Geophys. J. Int. 165, 149166.

Hornman, J.C. 2017. Field trial of seismic recording using distributed acoustic sensing with broadside sensitive fibre-optic cables, Geophys. Prospect., 65, 35-46.

Inbal, A., Ampuero, J.P. \& Clayton, R.W. 2016. Localized seismic deformation in the upper mantle revealed by dense seismic arrays, Science, $\mathbf{3 5 4}$, 88-92.

Inbal, A., Clayton, R.W. \& Ampuero, J.P. 2015. Imaging widespread seismicity at midlower crustal depths beneath Long Beach, CA, with a dense seismic array: Evidence for a depth-dependent earthquake size distribution, Geophys. Res. Lett. 42, 6314-6323.

Laboso, R.C. \& Davatzes, N.C. 2016. Fault-controlled damage and permeability at the Brady Geothermal System, Nevada, USA (SGP-TR-209), 41st Workshop on Geothermal Reservoir Engineering, Stanford University, Stanford, CA.

Lindsey, N.J., Martin, E.R., Dreger, D.S., Freifeld, B., Cole, S., James, S.R. \& Ajo-Franklin, J.B., 2017. Fiber-optic network observations of earthquake wavefields, Geophys. Res. Lett., 44, 11792-11 799.

Li, Z., Peng, Z., Hollis, D., Zhu, L. \& McClellan, J. 2018. High-resolution seismic event detection using local similarity for large-N arrays, Sci. Rep., 8, 1646, doi:10.1038/s41598-018-19728-w.
Li, Z., Peng, Z., Meng, X., Inbal, A., Xie, Y., Hollis, D. \& Ampuero, J. 2015. Matched Filter detection of microseismicity in Long Beach with a 5200-station dense array, in SEG Technical Program Expanded Abstracts 2015, pp. 2615-2619.

Martin, E.R., Castillo, C.M., Cole, S., Sawasdee, P.S. \& Yuan, S. 2017. Seismic monitoring leveraging existing telecom infrastructure at the SDASA: Active, passive, and ambient-noise analysis, Leading Edge, 36, 10251031.

Mateeva, A., Lopez, J., Mestayer, J., Wills, P., Cox, B., Kiyashchenko, D. \& Grandi, S. 2013. Distributed acoustic sensing for reservoir monitoring with VSP. Leading Edge, 32, 1278-1283.

Meng, X., Peng, Z. \& Hardebeck, J. 2013. Seismicity around Parkfield correlates with static shear stress changes following the $2003 M_{\mathrm{w}} 6.5$ San Simeon earthquake. J. geophys. Res., 118(7), 3576-3591.

Nathwani, J., Majer, E., Boyle, K., Rock, D., Peterson, J. \& Jarpe, S. 2011. DOE real-time seismic monitoring at enhanced geothermal system sites, in Thirty-sixth Workshop on Geothermal Reservoir Engineering, Stanford University, Stanford, CA.

Peng, Z. \& Zhao, P. 2009. Migration of early aftershocks following the 2004 Parkfield earthquake, Nat. Geosci., 2, 877-881.

Riahi, N. \& Gerstoft, P. 2015. The seismic traffic footprint: tracking trains, aircraft, and cars seismically. Geophys. Res. Lett., 42, 2674-2681.

Shapiro, S.A., 2015. Fluid-Induced Seismicity, Cambridge Univ. Press.

Shapiro, S.A., Rothert, E. \& Rath, V. 2005. Characterization of hydraulic properties of rocks using probability of fluid-induced microearthquakes, Geophysics. 70(2), F27-F33.

Shelly, D.R., Beroza, G.C. \& Ide, S. 2007. Non-volcanic tremor and lowfrequency earthquake swarms, Nature 446, 305-307.

Siler, D.L., Hinz, N.H., Faulds, J.E. \& Queen, J., 2016. 3D analysis of geothermal fluid flow favorability: Brady's, Nevada, USA, in 4lst Workshop on Geothermal Reservoir Engineering, Stanford University, Stanford, CA.

Turin, G.L. 1960. An introduction to matched filters, IRE Trans. Inf. Theory, 6(3), 311-329.

Wang, H.F., Zeng, X., Miller, D.E., Fratta, D., Feigl, K.L., Thurber, C.H. \& Mellors, R.J. 2018. Ground motion response to a ML 4.3 earthquake using co-located distributed acoustic sensing and seismometer arrays, Geophys. J. Int., 213, 2020-2036.

Zeng, X., Lancelle, C., Thurber, C., Fratta, D., Wang, H.F., Lord, N., .Chalari, A \& Clarke, A., 2017. Properties of ambient noise cross-correlation functions obtained from a distributed acoustic sensing array at Garner Valley, California, Bull. Seism. Soc. Am., 107, 603-610.

\section{SUPPORTING INFORMATION}

Supplementary data are available at $G J I$ online.

Figures S1-S4. DAS waveforms for four catalogued earthquakes.

Figure S5. Cross correlations of representative examples at different levels of visibility.

Figure S6. Comparison in the timings of the detected events and the vibroseis signals.

Table S1. Earthquake catalogue in the Brady geothermal field by template matching.

Please note: Oxford University Press are not responsible for the content or functionality of any supporting materials supplied by the authors. Any queries (other than missing material) should be directed to the corresponding author for the paper. 